\title{
Inductive Detection of Magnetostrictive Resonance
}

\author{
Jang-ik Park, SangGap Lee and Insuk Yu* \\ School of Physics and Nano-Systems Institute (NSI-NCRC), \\ Seoul National University, Seoul 151-747, South Korea \\ Yongho $\mathrm{Seo}^{\dagger}$ \\ Department of Nano Science \& Technology, \\ Sejong University, Seoul 143-747, Korea
}

(Dated: September 3, 2018)

\begin{abstract}
We have developed an inductive method to detect the magnetostrictive resonance signal and applied it to an ultrasonic magnetostrictive transducer sample. Slab shaped ferrite samples are mounted in an RF coil and actuated by pulse modulated RF magnetic field. A DC magnetic field is also applied and the resonance signal from the sample is detected by the same coil after the RF field is turned off. The detector system is similar to a conventional pulse NMR system with quadrature detection. The detected signal is sensitive to the bias DC field strength and direction as well as the dimension of the sample.
\end{abstract}

PACS numbers: 72.55.+s, 75.80.+q, 75.60.-d, 75.60.Ch

\footnotetext{
* corresponding author: isyu@snu.ac.kr

$\dagger$ corresponding author: yseo@sejong.ac.kr
} 


\section{INTRODUCTION}

To study elastic properties of solid materials, a pulse resonance technique composed of electromagnetic excitation and induction signal detection has been used widely. [1, 2]. Especially, for piezoelectric materials, Choi and Yu developed an piezoelectric resonance technique to study ferroelectric properties. 3] For magnetic materials, a coupling between electromagnetic field and sample magnetization mediates the excitation and detection. Possible sources for this coupling are the Lorentz force, the magnetization force, and the magne-

tostriction force. 4] The magnetostriction [5, 6, 7] phenomena originated from elastic domain wall motion was used for an oscillator first by Pierce[8]. This phenomenon has been used for actuators and sensors by oscillating it and measuring the frequency of elastic vibrations accurately. A representative example is magnetostrictive transducers converting electrical energy into mechanical energy. The magnetostriction transducers have been applied for ultrasonic sound generators, magnetostrictive optical wavelengths tuning, and acoustic delay lines [9]. Recently, highly magnetostrictive materials are investigated for practical application such as iron rare-earth compounds of terfenol-d [10] or ferromagnetic oxide composites [11].

Lanotte et al. 12, 13] had studied widely the bias field dependency of the amplitude and frequency of the acoustic waves in ferromagnetic materials using pulsed electromagnetic excitation. They used exciting and pickup coils with several hundred turns. The magnetoelastic wave amplitude was measured as a function of the exciting pulse burst frequency in the range of $2 \sim 120 \mathrm{kHz}$. In a similar method, the electromagnetic acoustic transducer (EMAT) technique has been used for detecting acoustoelastic stress, attenuation coefficient, grain size of metals, and magnetostriction coefficients [4, 14, 15, 16, 17]. For EMAT technique, a ferromagnetic sample is located beneath of a meander-line which is beneath of a permanent magnet or a disk shape sample is located in a solenoid coil. The solenoid coil is used for applying constant bias magnetic field along the sample axis and the meander-line coil is to induce the dynamic field in the circumferential direction through magnetostrictive effect and receive the shear wave through the reversed magnetostrictive effect.

In this paper, we report the rf pulse type resonance detection method for magnetoacoustic wave in a slab shape ferrite material used for an ultrasonic magnetostrictive transducer. The unique features of our method are single impulse excitation and Fourier transformation detection. 


\section{EXPERIMENTS}

Overall experimental setup is similar to a pulse type NMR spectrometer and the sample is a commercially available magnetostrictive transducer ferrite material commonly used for an ultrasonic cleaner produced by TDK corp. [19]

A schematics of the experimental spectrometer setup used in our experiment is shown in Fig. 1. This setup is similar to a conventional pulsed NMR spectrometer with a series tuned sample coil and quadrature detection receiver [3, 20]. The setup consists of the power amplifier which applies a pulse modulated high voltage RF magnetic field to the sample inside the sample coil and the quadrature detection receiver which picks up the resonance signal from the sample. The sample and sample coil are placed inside an electromagnet which apply the static magnetic field to the sample. The signal from the sample is transmitted to an analog-digital board in a PC with 8 bit voltage resolution and 1024pt digitization for each channel. The sample coil we used is a solenoid of $25 \mathrm{~mm}$ diameter and $10 \mathrm{~mm}$ height in 11 turns tightly wound. As shown in the Fig. 1, the dc bias magnetic field is perpendicular to the pulsed RF magnetic field. When we applied the static field parallel to the RF field, no signal was found.

The ferrite samples were cut into various sizes using a diamond saw. The samples are slab shape of $\sim 1 \mathrm{~mm}$ thickness, $\sim 10 \mathrm{~mm}$ width, and $\sim 50 \mathrm{~mm}$ length. A supporting structure made by thin teflon sheet clamps one end of the sample. The sample is rotatable by the teflon support. The spectrometer is tuned to $5 \mathrm{MHz}$ and the pulses of width $0.5 \mu \mathrm{s}$ and repetition time $10 \mathrm{~ms}$ are applied throughout this work.

\section{RESULTS AND DISCUSSION}

The detected signal from the slab shape sample of $0.79 \mathrm{~mm} \times 9.06 \mathrm{~mm} \times 42.85 \mathrm{~mm}$ is shown in Fig. 2. The signal is 64 times sampled to average out noise. The bias magnetic field intensity is $0.6 \mathrm{kG}$ and applied parallel to the sample surface. Figure 2 shows the ring-down signal, when the sample is parallel to magnetic field $\left(\theta=0^{\circ}\right)$. The inset of Fig. 2 is the Fourier transformed (FT) waveform of the signal. The several peaks in the spectrum are shown with equal spacing. The series of peaks has frequencies

$$
f^{(n)}=\frac{n c}{2 d}
$$


where $n$ is positive integer, $c$ acoustic wave velocity, $d$ length of the acoustic wave. 17] The highest peak corresponds to 12 th harmonic $\left(f^{(12)}=4.84 \mathrm{MHz}\right)$ and the frequency spacing $\delta f=0.403 \mathrm{MHz}$.

We investigate the dependency of resonance frequency on the sample geometry. The magnetic field is applied parallel to the sample surface and kept to $0.6 \mathrm{kG}$. After the resonance signal of a sample is measured, the sample is dismounted to narrow the width by cutting it with a diamond saw. The frequency separation between the peaks is shown as a function of sample width in Fig. 3(a). The error bar comes from the fact that we have only 3 or 4 resonance peaks in the spectrum. The graph shows the inverse proportionality between the width $W$ of sample and the frequency separation $\delta f$ of harmonics peaks, as

$$
\delta f \propto \frac{1}{W} .
$$

Judging from this dependency, the acoustic waves are travelling in width direction and possible mode is flexural mode. Therefore, $d$ in Eq. (11) is equal to $W$ and the acoustic wave velocity $c$ is estimated as $7.31 \times 10^{3} \mathrm{~m} / \mathrm{s}$.

Next we inspect the sample thickness dependence of the resonance signal. Samples are prepared with $9.06 \mathrm{~mm}$ width, $42.85 \mathrm{~mm}$ length and various thicknesses. The magnetic field is applied parallel to the sample surface and kept to $0.6 \mathrm{kG}$, as the same as for previous measurement. Fig. 3(b) shows the dependence of resonance frequency on the sample thickness. Because the acoustic waves are travelling in width direction, the frequency should not depend on thickness. Real data shows a slight dependency on thickness. We attribute the change of the resonance frequency to change of the internal strain influenced by size effect.

The resonance frequency and amplitude are measured as functions magnetic field $H$, as shown in Fig. 4 (a) and (b), respectively. The dimension of this sample is $0.79 \times 9.06$ $\times 42.85 \mathrm{~mm}^{3}$ and the bias field is applied parallel to the sample surface. Among many peaks in Fourier transformed signal, the highest peak frequency is shown in Fig. 4(a) The increase of the resonance frequency depending on the bias field reflects the increase of stiffness of the sample. The inset in Fig. 4(a) shows the separation $\delta f$ between the harmonics of resonance frequency versus the bias field dependence. The harmonics of resonance frequency are measured in our measurable spectral range between $4 \mathrm{MHz}$ and $6 \mathrm{MHz}$. These data show a tendency that resonance frequencies grows rapidly at low magnetic field and becomes saturated in high magnetic field. This looks a typical behavior of a magnetization change 
versus external field. Therefore, the resonance frequency shift is affected by a mechanical strain induced by the bias field. Similar behaviors on the other kinds of magnetic samples were reported by Lanotte, et al, which was explained as Young's modulus change $(\Delta E$ effect). 12, 13] No noticeable hysteresis is found in the resonance frequency change during increasing and decreasing the bias magnetic field.

As shown in Fig. 4(b), the dependence of the amplitude on $H$ shows a peak behavior at $H=0.7 \mathrm{kG}$. Similar behaviors were reported in many literatures. 4, 12, 18] Thompson explained this peak behavior as magnetostrictive contribution. We also attribute it to the field dependency of magnetostriction coefficient. As $H$ increases, magnetic domains begin to move against its stable configuration. When $H$ is larger than its saturation field, the domain wall motion will disappear. Therefore, for the saturation magnetic field, the $E$ and $f$ do not change anymore and $A$ decreases down to zero.

The $f$ and $A$ are measured, as the bias field direction is varied by rotating the teflon support holding the sample. The sample dimension is the same as above and the bias magnetic field is $0.6 \mathrm{kG}$. The resonance frequency and amplitude change versus the angle $(\theta$ as shown in Fig. (1)) between the bias field and sample surface are shown in Fig. 5(a) and (b), respectively. The inset of the Fig. 5(a) shows the $\delta f$ between the harmonic frequencies within our spectral range from $4 \mathrm{MHz}$ to $6 \mathrm{MHz}$. This experiment has no hysteresis as increasing or decreasing on angle also. We attribute the angle dependency to the sample geometry. Because the sample is a slab shape, it has magnetic anisotropy and domain walls have preferential direction. The $\theta$ increase can be considered as an effective magnetic field decrease. Therefore, the $f$ and $A$ decrease as if they are changed by the $H$ decrease as shown in Fig. 4.

In summary, we report a new experimental instrument using pulse NMR spectrometer to study the magneto-elastic acoustic wave in ferromagnetic materials. Our pulse modulated inductive detection technique combined with Fourier transform analysis has an advantage measuring the resonance spectrum at a single pulse modulated rf excitation.

\section{Acknowledgments}

This work is supported by the National Core Research Center program of the Korea Science and Engineering Foundation (KOSEF) through the NANO Systems Institute of 
Seoul National University.

[1] J. L. Davis, Wave Propagation in Solids and Fluids (springer-Verlag, New York, 1988).

[2] W. P. Mason, Physical Acoustics (Academic Press, New York, 1964).

[3] K. Choi and I. Yu, Rev. Sci. Instrum. 60, 3249 (1989).

[4] H. Ogi, J. Appl. Phys. 82, 3940 (1997).

[5] R. M. Bozorth, E. F. Tilden, and A. J. Williams, Phys. Rev. 99, 1788 (1955).

[6] R. M. Bozorth and J. G. Walker, Phys. Rev. 88, 1209 (1952).

[7] J. C. Slonczewski, Phys. Rev. 122, 1367 (1961).

[8] G. W. Pierce, Proc. Am. Acad. Sci. 63, (1928).

[9] E. Hristoforou, and R. E. Reilly, IEEE Transactions on Magnetics 26, 1563 (1990).

[10] R. Chung, R. Weber, and D. C. Jiles, IEEE Transactions on Magnetics 27, 5358 (1991).

[11] L. H. Chen, T. J. Klemmer, H. Mavoori, and S. Jin, Appl. Phys. Lett. 74, 2047 (1999).

[12] L. Lanotte, C. Luponio, and F. Porreca, J. Appl. Phys. 50, 438 (1979).

[13] L. Lanotte, C. Luponio, and F. Porreca, J. Appl. Phys. 69, 7735 (1991).

[14] K. Kawashima and O. B. Wright, J. Appl. Phys. 72, 4830 (1992).

[15] M. Hirao, H. Ogi, and H. Fukuoka, Rev. Sci. Instrum. 64, 3198 (1993).

[16] M. Hirao and H. Ogi, Appl. Phys. Lett. 64, 2217 (1994).

[17] H. Ogi, M. Hirao, and S. Aoki J. Appl. Phys. 90, 438 (2001).

[18] R. B. Thompson, IEEE Trans. Sonics Ultrasonic SU-25, 7 (1978).

[19] Ferrite magnetostrictive material (V2XП20), produced by TDK co. in Japan.

[20] A. Abragam, The principle of Nuclear Magnetism, Oxford University Press (1961). 
FIG. 1: Experimental setup is shown. The spectrometer is tuned at $5 \mathrm{MHz}$ and has quadrature detection receiver. The ferrite sample is located inside the electromagnet. The sample can be rotated manually.

FIG. 2: Ring down signal generated from a $0.79 \mathrm{~mm} \times 9.06 \mathrm{~mm} \times 42.85 \mathrm{~mm}$ sample. It is measured by an oscilloscope with 1024 sampling points and 8-bit voltage resolution. The inset shows the Fourier transform of the signal. In the Fourier transform process, we exclude the first hundred points to reduce the secondary effect from the preamplifier.

FIG. 4: Bias field $H$ affects the resonance frequency $f(\mathrm{a})$ and the signal amplitude $A$ (b). The inset shows the dependency of $\delta f$ on the bias field.

FIG. 5: The bias field direction on the sample affects the resonance frequency (a) and the signal amplitude (b). $\theta$ indicates the angle between the sample and the bias field. The inset shows the dependency of $\delta f$ on the field direction.

FIG. 3: (a) The frequencies of harmonic resonance peaks depend on the sample width. The spacing of frequencies is shown as a function of the inverse of width. The samples are located parallel to the bias field $0.6 \mathrm{kG}$. $(\theta=0$.) (b) The dependency of resonance frequency on the sample thickness is shown. This dependency is due to the change of internal strain influenced by size effect. 